\title{
Selective modification of the unquenched orbital moment of manganese introduced by carbon dopant in epitaxial $\mathrm{Mn}_{5} \mathrm{Ge}_{3} \mathrm{C}_{0.2} / \mathrm{Ge}(111)$ films
}

\author{
R. Kalvig $\odot,{ }^{1}$ E. Jedryka $\odot,{ }^{1}$ M. Wojcik $\odot,{ }^{1}$ M. Petit $\odot,{ }^{2}$ and L. Michez $\odot^{2}$ \\ ${ }^{1}$ Institute of Physics, Polish Academy of Sciences, Aleja Lotników 32/46, PL-02668 Warsaw, Poland \\ ${ }^{2}$ Aix Marseille Univ, CNRS, CINAM, Marseille, France
}

(Received 3 September 2019; revised manuscript received 24 January 2020; accepted 7 February 2020; published 2 March 2020)

\begin{abstract}
${ }^{55} \mathrm{Mn}$ NMR was used to investigate the effect of carbon doping on the local magnetic anisotropy in $\mathrm{Mn}_{5} \mathrm{Ge}_{3}$ epitaxial films (space-group $P 6_{3} / \mathrm{mcm}$ ). It was found that carbon enters interstitially in the vicinity of the $6(\mathrm{~g})$ crystallographic positions, occupying the $2(b)$ octahedral voids. The magnetic properties of the Mn atoms located in the corners of a host octahedron are strongly modified by the presence of carbon. Their magnetic moment is reduced by $0.7 \mu_{B}$ with respect to the pristine $\mathrm{Mn}_{5} \mathrm{Ge}_{3}$ film and the anisotropy of their orbital moment, measured as a difference between its value along the hexagonal $c$ direction and on the $c$ plane is reduced to $0.058 \mu_{B}$, whereas, in the pristine $\mathrm{Mn}_{5} \mathrm{Ge}_{3}$ films, it oscillated every $60^{\circ}$ between $0.151 \mu_{B}$ and 0 . These changes are responsible for a significant decrease in magnetocrystalline anisotropy, which was reported to drop by an order of magnitude upon doping the $\mathrm{Mn}_{5} \mathrm{Ge}_{3}$ films with carbon.
\end{abstract}

DOI: 10.1103/PhysRevB.101.094401

\section{INTRODUCTION}

$\mathrm{Mn}_{5} \mathrm{Ge}_{3}$ is a metallic ferromagnet crystallizing in the hexagonal $\mathrm{D} 8_{8}$ structure (space-group $P 6_{3} / \mathrm{mcm}$ ). This compound has been intensively studied as a potential candidate for spintronic applications, following reports that thin films of $\mathrm{Mn}_{5} \mathrm{Ge}_{3}$ can be epitaxially grown on $\mathrm{Ge}(111)$ substrates with atomically sharp interface [1], making it a potential source of polarized carriers into Ge via electron tunneling through the Schottky barrier with no need of an insulating barrier despite the lattice mismatch of 3.7\% [2-4]. A high spin polarization of the conduction electrons at the Fermi level has been predicted theoretically [5] and confirmed experimentally [6]. Moreover, this compound presents a strong uniaxial magnetocrystalline anisotropy along the hexagonal $c$ axis [7-9] opening a possibility to combine spintronics with the data storage [10].

A pristine $\mathrm{Mn}_{5} \mathrm{Ge}_{3}$ compound reveals the Curie temperature of $296 \mathrm{~K}$, which is rather low for practical applications, but it was shown that it can be significantly increased by doping with carbon [11]. Epitaxial growth of $\mathrm{Mn}_{5} \mathrm{Ge}_{3} \mathrm{C}_{x}$ films with good crystal quality has been demonstrated for carbon content up to around $x=0.6$, boosting the Curie temperature to $430 \mathrm{~K}$ [12]. The unit cell of $\mathrm{Mn}_{5} \mathrm{Ge}_{3}$, shown in Fig. 1, contains two formula units with $\mathrm{Mn}$ atoms located in two crystallographic positions: $4(d)$ (further denoted as $\mathrm{Mn}_{I}$ sites) and $6(\mathrm{~g})$ (denoted as $\mathrm{Mn}_{I I}$ sites). It has been shown that the structure remains unchanged when carbon is added [8] and it is assumed that carbon enters the octahedral voids created by the $\mathrm{Mn}_{I I}$ atoms [11]. This suggestion is supported by a calculation of the formation energy of alternative site occupancies (three substitutional positions in addition to the above-mentioned voids) [12]. However, the experimental confirmation of such a carbon location has been missing and will be provided by the ${ }^{55} \mathrm{Mn}$ Nuclear magnetic resonance (NMR) experiments described in this paper.

In addition to boosting the critical temperature, the carbon admixture improves the thermal structural stability of $\mathrm{Mn}_{5} \mathrm{Ge}_{3}$ thin films [14]. The enhancement of the magnetic properties has been explained by the onset of a $90^{\circ}$ ferromagnetic superexchange between the $\mathrm{Mn}_{I I}$ atoms mediated by carbon, enhancing the Mn-Mn interactions [15]. However, the presence of carbon has the drawback of a lowered magnetization, which has been attributed to a strong hybridization of carbon $2 p$ and manganese $3 d$ orbitals $[12,15]$. Moreover, the C-doped specimens reveal a decreased magnetocrystalline anisotropy: the critical thickness defining the out-of-plane orientation of magnetization and the onset of a stripe domain structure has been reported to increase from $20 \mathrm{~nm}$ in the pristine $\mathrm{Mn}_{5} \mathrm{Ge}_{3}$ to $30 \mathrm{~nm} \mathrm{Mn}_{5} \mathrm{Ge}_{3} \mathrm{C}_{0.7}$ [8,12]. The corresponding uniaxial magnetocrystalline anisotropy constant dropped by an order of magnitude from $5.7 \times 10^{6} \mathrm{erg} / \mathrm{cm}^{3}$ in $\mathrm{Mn}_{5} \mathrm{Ge}_{3}$ [9] to $6 \times 10^{5} \mathrm{erg} / \mathrm{cm}^{3}$ in $\mathrm{Mn}_{5} \mathrm{Ge}_{3} \mathrm{C}_{0.7}$ at $15 \mathrm{~K}$ [8].

The aim of this paper is to investigate the microscopic origin of the observed drop of anisotropy in the $\mathrm{Mn}_{5} \mathrm{Ge}_{3} \mathrm{C}_{x}$ films. As shown in the theoretical work of Bruno [16], the magnetocrystalline anisotropy in metals is associated with the unquenched orbital momentum, via spin-orbit interaction. In our previous paper on the pristine epitaxial films of $\mathrm{Mn}_{5} \mathrm{Ge}_{3}$, we have shown that NMR reveals a strong anisotropy of the ${ }^{55} \mathrm{Mn}$ hyperfine fields and suggested that this is due to the orbital contribution. Indeed, it can be readily shown that the orbital hyperfine field is the only component of the local field on the nucleus that can account for the anisotropy observed in the pristine and in the C-doped $\mathrm{Mn}_{5} \mathrm{Ge}_{3}$ films.

The NMR resonance frequency $v$ is determined by the effective magnetic-field $B_{\text {eff }}$ at the site of the nucleus under 


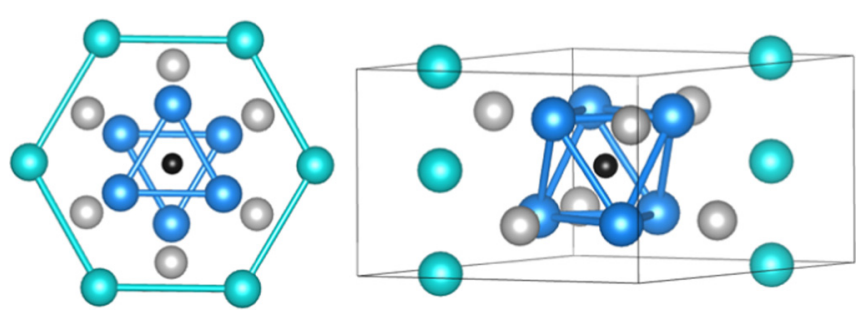

FIG. 1. Crystal structure of $\mathrm{Mn}_{5} \mathrm{Ge}_{3} \mathrm{C}$. Left: Top view on the hexagonal plane; right: Side view. Colored spheres denote, respectively: $\mathrm{Mn}_{I}$ (green), $\mathrm{Mn}_{I I}$ (blue), Ge (gray), and C (black). This drawing was prepared using the VESTA software [13].

study,

$$
v=\gamma B_{\text {eff }},
$$

where $\gamma$ is the gyromagnetic ratio. For the case of ${ }^{55} \mathrm{Mn} \gamma=$ $10.553 \mathrm{MHz} / \mathrm{T}$.

In the absence of an external magnetic field $\left(B_{\mathrm{ext}}=0\right)$, the effective field on the nucleus consists of the hyperfine field $B_{h f}$ due to the unpaired electrons of the same atom and the dipolar field $B_{\text {dip }}$ due to the magnetic moments of the surrounding atoms. When the external magnetic field is applied, the $B_{\text {ext }}$ component adds to the effective field on the nucleus,

$$
\vec{B}_{\text {eff }}=\vec{B}_{h f}+\vec{B}_{\text {dip }}+\vec{B}_{\text {ext }} .
$$

Let us, now, consider different constituents of the effective field in order to pinpoint the components that can possibly contribute to the overall anisotropy of the effective field.

The main component of the effective field $B_{\text {eff }}$ is the hyperfine field $B_{h f}$, consisting of the following contributions:

$$
\vec{B}_{h f}=\vec{B}_{h f}^{c f}+\vec{B}_{h f}^{\text {orb }}+\vec{B}_{h f}^{\text {dip }} .
$$

$\vec{B}_{h f}^{c f}$ is the isotropic contact Fermi term due to the nonzero density of the $s$ electrons at the site of the nucleus and generally consists of the following contributions:

$$
\vec{B}_{h f}^{c f}=\vec{B}_{h f}^{\text {core }}+\vec{B}_{h f}^{\text {cond }}+\vec{B}_{h f}^{\text {trans }},
$$

where $B_{h f}^{\text {core }}$ and $B_{h f}^{\text {cond }}$ are due to the spin polarization of the core and valence electrons by the on-site magnetic moment, respectively, whereas $B_{h f}^{\text {trans }}$ represents the contribution to the polarization of valence electrons by the magnetic moments of the surrounding atoms. Assuming a dominant contribution of the on-site magnetic moment, the transferred term can be neglected, and the Fermi contact term can be expressed as [17]

$$
\vec{B}_{h f}^{c f}=A_{c f} \vec{\mu}_{s},
$$

where $A_{c f}$ is the hyperfine interaction constant having a negative value (Fermi contact field is antiparallel to the spin magnetic moment $\vec{\mu}_{s}$ ). $\vec{B}_{h f}^{\text {dip }}$ is the field due to the spin magnetic moments of the unpaired electrons in the outer $d$ shells, and $\vec{B}_{h f}^{\text {orb }}$ is the anisotropic orbital contribution due to the unquenched orbital momentum and is directly proportional to the orbital moment $\mu_{l}[5,18]$,

$$
\vec{B}_{\text {orb }}=2 \mu_{B}\left\langle r^{-3}\right\rangle_{l} \vec{\mu}_{l}=A_{\text {orb }} \vec{\mu}_{l},
$$

where $\left\langle r^{-3}\right\rangle_{l}$ is the average expectation value of the operator $r^{-3}$ of the radial wave function of $3 d$ electrons and $\mu_{B}$ denotes the Bohr magneton. The corresponding hyperfine interaction constant $A_{\text {orb }}$ has a positive value. In the bulk systems, the dipolar part $B_{h f}^{\mathrm{dip}}$ of the hyperfine field $B_{h f}$ is negligibly small compared to the other two contributions and can be neglected. Indeed, it has been neglected in the first-principles calculations of hyperfine fields in $\mathrm{Mn}_{5} \mathrm{Ge}_{3}$ [5] where it was shown that the hyperfine field in this compound, calculated as a sum of the contact and orbital components, reproduces correctly the experimental values.

The second term of Eq. (2), the dipolar field $B_{\text {dip }}$ can be estimated by calculating the contribution of the discrete magnetic moments within a Lorentz sphere of a radius $r$ sufficiently large to secure the convergence of the $1 / r^{3}$ series. To the calculated field of the discrete moments, the Lorentz cavity field $B_{\text {Lor }}$ as well as the macroscopic demagnetizing field $B_{\mathrm{dem}}$ have to be added

$$
\vec{B}_{\text {dip }}=\vec{B}_{\text {discr }}+\vec{B}_{\text {Lor }}+\vec{B}_{\text {dem }} .
$$

To compute the dipolar field from the discrete magnetic moments, we have considered a Lorentz sphere with the radius of $35 \mathrm{~nm}$. We find that the dipolar discrete field $B_{\text {discr }}$ on the $\mathrm{Mn}_{I}$ site of the pristine $\mathrm{Mn}_{5} \mathrm{Ge}_{3}$ equals $-0.14 \mathrm{~T}$ along the $c$ direction, and the anisotropy between the $c$ direction and the $c$ plane is $-0.21 \mathrm{~T}$. In the $\mathrm{Mn}_{I I}$ site, the dipolar field along the $c$ direction is $-0.07 \mathrm{~T}$, whereas the anisotropy between the $c$ direction and the $c$ plane oscillates with a period of $60^{\circ}$ with a maximum amplitude of $-0.19 \mathrm{~T}$. These values are, at least, one order of magnitude smaller than the anisotropy of hyperfine fields experimentally observed in this material (1.52 $\mathrm{T}$ in the $\mathrm{Mn}_{I}$ site and 4.08 $\mathrm{T}$ in the $\mathrm{Mn}_{I I}$ site, respectively). In the case of $\mathrm{Mn}_{5} \mathrm{Ge}_{3} \mathrm{C}_{0.2}$, accounting for the modified magnetic moment of Mn, calculated in Ref. [15], we obtain similar insignificant values of dipolar fields and their anisotropy. Bearing in mind that the Lorentz cavity field is a constant, not contributing to the anisotropic effects, the only term of the dipolar field (7) that has to be accounted for is the demagnetizing field, and this has been performed in the present paper by extrapolating the $v(B)$ plot to the zero external field (see Figs. 9 and 12). In view of the above considerations, the only component of the effective field $B_{\text {eff }}$ accountable for the observed anisotropy is the orbital term of hyperfine field.

In this paper, we first investigate the effect of carbon on the magnitude of hyperfine fields and on the local magnetic moment in different Mn sites by studying ${ }^{55} \mathrm{Mn}$ NMR at zero field and in a strong external magnetic field applied perpendicular to the film plane. As a second step, we apply the magnetic field in different orientations within the film plane to study the hyperfine field anisotropy $\Delta B=B(M \|$ [001])- $B[M \|(001)]$. We find that the primary effect of carbon concerns the $\mathrm{Mn}_{I I}$ atoms that are located in the corners of those octahedra that happen to host the $\mathrm{C}$ dopant. Those $\mathrm{Mn}_{I I}$ sites experience about a $20 \%$ drop of the magnetic moment and a strongly modified anisotropy of hyperfine fields. On the other hand, the $\mathrm{Mn}_{I I}$ atoms that do not have carbon as a nearest neighbor (nn) are practically unaffected by the presence of carbon, retaining the same anisotropy as the $\mathrm{Mn}_{I I}$ sites in the pristine film. A moderate modification of hyperfine fields on the $\mathrm{Mn}_{I}$ sites (via the transferred field from the 
altered $\mathrm{Mn}_{I I}$ magnetic moments) confirms the local selective effect of carbon on magnetic properties of $\mathrm{Mn}_{5} \mathrm{Ge}_{3}$ films.

\section{EXPERIMENTAL DETAILS}

A 300-nm-thick $\mathrm{Mn}_{5} \mathrm{Ge}_{3} \mathrm{C}_{0.2}$ thin film was synthesized on a $\mathrm{Ge}(111)$ substrate in a conventional ultra-high-vacuum molecular beam epitaxy setup with a base pressure of $3 \times$ $10^{-8} \mathrm{~Pa}$. The entire growth process was monitored in situ by reflection high-energy electron-diffraction (RHEED) measurements. Prior to the sample deposition, a 100-nm-thick Ge buffer was epitaxially grown on the chemically cleaned and thermally flashed substrate. This essential step ensured a high structural quality of the starting surface as shown in the RHEED pattern of Fig. 1(a) of Ref. [2]. The substrate had a rectangular shape with one edge along the [1111] crystallographic direction and the other edge along the [112] direction of $\mathrm{Ge}$.

The sample was then synthesized using the room temperature co-deposition method that has been described in detail in Ref. [2]. Briefly, this technique consists of the coevaporation of $\mathrm{Mn}, \mathrm{Ge}$, and $\mathrm{C}$. The flux rates of the three elements were beforehand carefully tuned with respect to the desired stoichiometry. The C-doped $\mathrm{Mn}_{5} \mathrm{Ge}_{3}$ phase was formed by nucleation as soon as the atoms reached the surface with a growth direction along the $c$ axis. No other phase was detected with the RHEED measurements. This result was then confirmed by postgrowth ex situ measurements. The singlephase film is fully epitaxial and of very high crystalline quality as demonstrated by x-ray diffraction (Fig. 4 of Ref. [2]) and transmission electron microscopy (Fig. 3 of Ref. [2]) characterization. The epitaxial relationship between the Ge substrate and the $\mathrm{Mn}_{5} \mathrm{Ge}_{3}$ film is as follows: $\mathrm{Mn}_{5} \mathrm{Ge}_{3}(001) \| \mathrm{Ge}(111)$ and [010] of $\mathrm{Mn}_{5} \mathrm{Ge}_{3} \|$ [112̄] of $\mathrm{Ge}$ [2].

${ }^{55} \mathrm{Mn}$ NMR spectra were recorded at $4.2 \mathrm{~K}$ with an automatic broadband phase-sensitive spin-echo spectrometer [19]. To perform the experiments in the presence of the magnetic field, the probe was placed in a superconducting magnet working up to 6 T. For zero-field experiments, the untuned broadband probe was used, whereas the in-field experiments were performed with the use of a probe that was automatically tuned to resonance frequency. At each external field value, several spectra have been recorded at different amplitudes of the rf excitation field. The optimum amplitude of the spin-echo signal as well as the NMR enhancement factor at each frequency have been computed. This procedure-called the Panissod protocol-makes it possible to account for the frequency variation of the enhancement factor and correct the NMR signal intensity [20].

\section{RESULTS AND DISCUSSION}

\section{A. ${ }^{55} \mathrm{Mn}$ hyperfine fields in different $\mathrm{Mn}$ sites of $\mathrm{Mn}_{5} \mathrm{Ge}_{3} \mathrm{C}_{0.2}$-modification due to carbon}

Figure 2 presents the ${ }^{55} \mathrm{Mn}$ NMR spectra recorded in the absence of an external magnetic field from the two epitaxial films with thickness of $300 \mathrm{~nm}$ : (a) pristine $\mathrm{Mn}_{5} \mathrm{Ge}_{3}$ and (b) carbon-doped film with nominal composition $\mathrm{Mn}_{5} \mathrm{Ge}_{3} \mathrm{C}_{0.2}$. The zero-field NMR spectrum from the pristine sample [Fig. 2(a)] has been discussed in detail in our previous paper

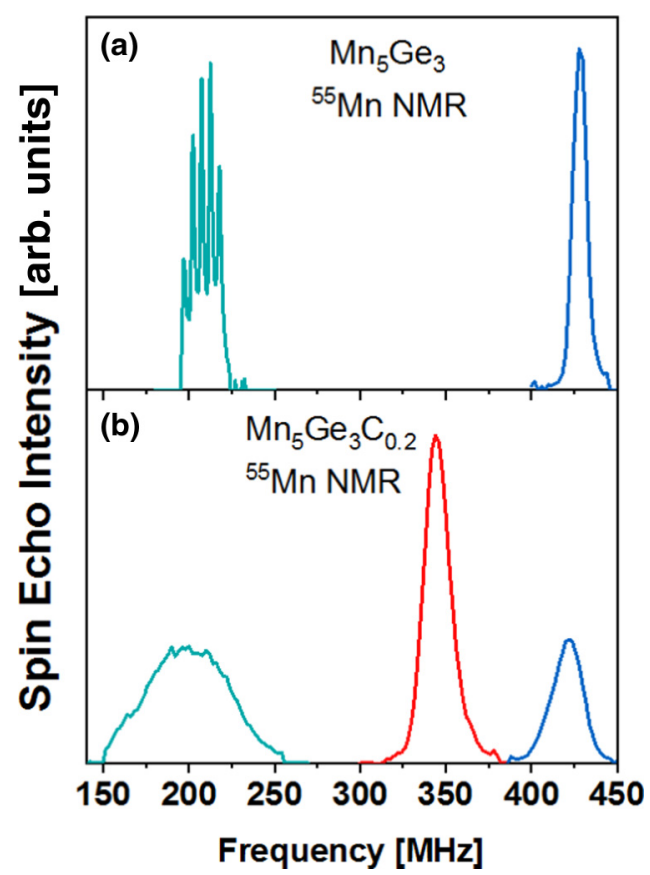

FIG. 2. Zero-field ${ }^{55} \mathrm{Mn}$ NMR spectra recorded at $4.2 \mathrm{~K}$ from the 300-nm films of $\mathrm{Mn}_{5} \mathrm{Ge}_{3}$ and $\mathrm{Mn}_{5} \mathrm{Ge}_{3} \mathrm{C}_{0.2}$ epitaxially grown on the $\mathrm{Ge}(111)$ substrate. Green: The NMR line from the $\mathrm{Mn}_{I}$ sites; blue: $\mathrm{Mn}_{I I}$ sites with no carbon nearest neighbors; and red: $\mathrm{Mn}_{I I}$ sites having carbon nearest neighbors.

[21] where it was shown that the two NMR lines observed in distinctly different frequency ranges represent manganese in the two nonequivalent sites in the crystal lattice: Mn located in the $4(d)$ sites (later denoted as $\mathrm{Mn}_{I}$ ) gives rise to the quadrupole-split line centered at the frequency of $207.5 \mathrm{MHz}$, whereas the $6(\mathrm{~g})$ sites (denoted as $\mathrm{Mn}_{I I}$ ) contribute to the single NMR line centered at $428 \mathrm{MHz}$ [21].

Comparing the ${ }^{55} \mathrm{Mn}$ NMR spectrum from the carbondoped sample presented in Fig. 2(b) to that of the pristine film, three effects are readily visible: (i) The NMR line corresponding to the $\mathrm{Mn}_{I}$ sites does not reveal the quadrupolar structure and becomes significantly broadened with gravity center shifted towards lower frequencies, (ii) the $\mathrm{Mn}_{I I} \mathrm{NMR}$ line is much less intensive, whereas its half-width is increased, and the line center is shifted from 428 to $420 \mathrm{MHz}$, and (iii) a satellite line appears around $344 \mathrm{MHz}$ - it is much more intensive than the original $\mathrm{Mn}_{I I}$ signal and has a similar linewidth. To identify the origin of the observed NMR lines, we first need to consider the orientation of magnetization giving rise to the NMR signal in a zero external magnetic field. It is common knowledge that the NMR signal is strongly enhanced within the domain walls in which case, the NMR line shape integrates the contributions from magnetic moments oriented in all the crystallographic directions enveloped by the wall profile. On the other hand, the domain's interior gives the NMR signal determined by the orientation of spins inside the magnetic domain. Prevalence of one of these two contributions depends on the domain-wall width and mobility as well as on the signal excitation conditions.

Our previous study of a pristine $\mathrm{Mn}_{5} \mathrm{Ge}_{3}$ film has unambiguously shown that the zero-field ${ }^{55} \mathrm{Mn}$ NMR signal in the 

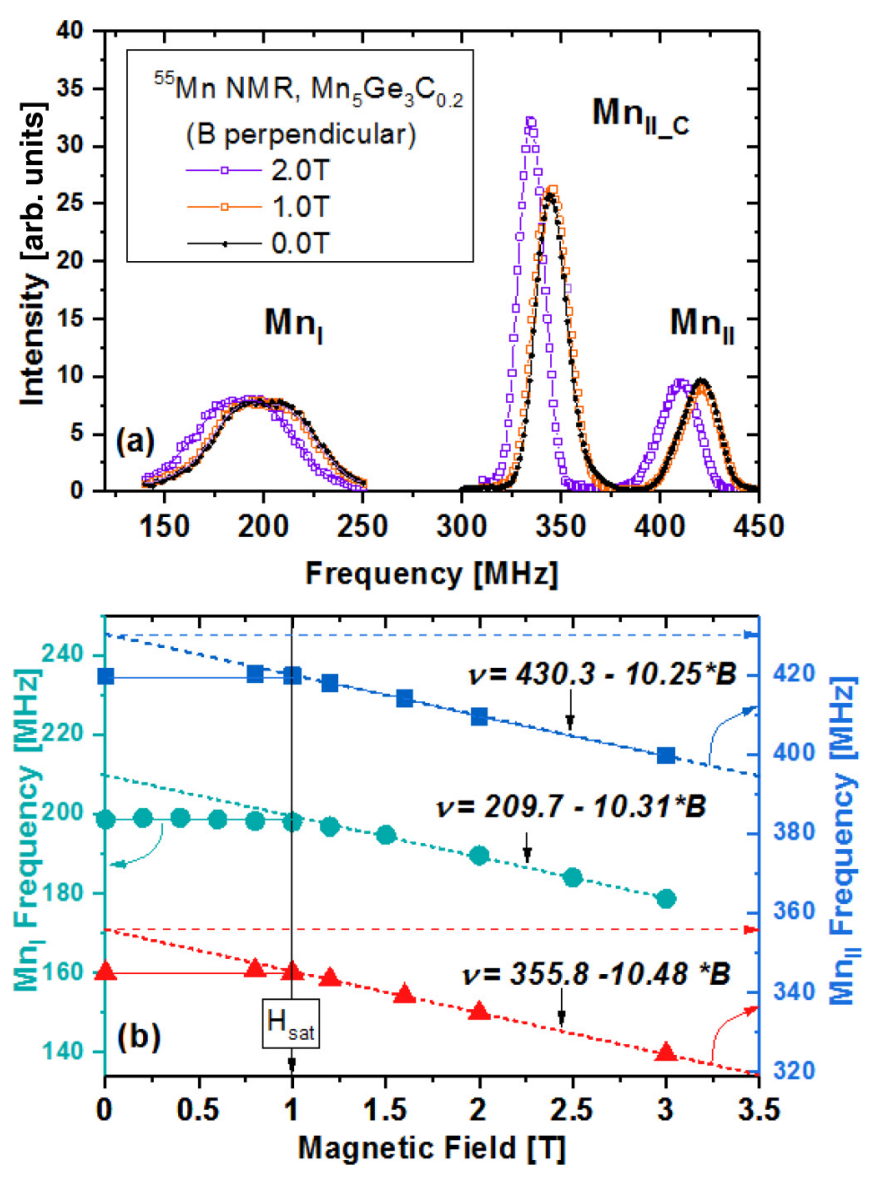

FIG. 3. (a) ${ }^{55} \mathrm{Mn}$ NMR spectra recorded at $4.2 \mathrm{~K}$ from the $\mathrm{Mn}_{5} \mathrm{Ge}_{3} \mathrm{C}_{0.2}$ film at different values of the external magnetic field oriented perpendicular to the film plane; (b) ${ }^{55} \mathrm{Mn}$ NMR frequency in $\mathrm{Mn}_{5} \mathrm{Ge}_{3} \mathrm{C}_{0.2}$ as a function of the out-of-plane field: Left scale: $\mathrm{Mn}_{I}$ crystalline sites; right scale: $\mathrm{Mn}_{I I}$ crystalline sites. The colors of the symbols correspond to particular NMR lines as defined in Fig. 2.

300-nm film originates from the magnetic domains with magnetization oriented perpendicular to the film plane, i.e., along the hexagonal $c$ axis [21]. In order to verify whether this remains true also in the case of the C-doped sample, we performed the NMR experiment in the presence of an external magnetic field applied perpendicular to the film plane. Figure 3(a) shows the NMR spectra recorded from the 300-nm $\mathrm{Mn}_{5} \mathrm{Ge}_{3} \mathrm{C}_{0.2}$ film at three different magnetic-field values, before and after the magnetic saturation in the outof-plane direction, whereas Fig. 3(b) shows the frequency position of the respective line center as a function of the magnetic-field strength. Clearly, the line shape and the frequency position of the entire NMR spectrum do not change until the magnetic saturation in the $c$ direction is reached (i.e., around $1 \mathrm{~T}$ ), showing that the effect of the external magnetic field is screened by the domain structure.

For the field values above the saturation field, the spectrum shifts uniformly towards lower frequencies with a negative slope corresponding to the ${ }^{55} \mathrm{Mn}$ gyromagnetic ratio whereas the shape of the NMR line remains unchanged. This behavior is analogous to that observed in the pristine sample [21]. The negative slope after saturation means that the dominant contribution to the Mn hyperfine field is due to the Fermi contact term Eq. (5), which is proportional to the local magnetic moment. On the other hand, the invariance of the spectrum's shape in the presence of the out-of-plane field confirms that the zero-field NMR spectrum in the C-doped film originates from the regions within the sample where magnetization is perpendicular to the film plane (i.e., parallel to the crystallographic $c$ direction).

We can, thus, conclude that the satellite line observed at $344 \mathrm{MHz}$ originates also from the $c$ direction and is a fingerprint of new manganese environments created as a result of incorporation of carbon atoms in the vicinity of the $6(\mathrm{~g})$ lattice sites. These most probably enter the $2(b)$ voids formed by the $\mathrm{Mn}_{I I}$ octahedra. Such a location has been previously inferred from the crystallographic studies of this compound and comparison with other similar Nowotny phases [22]. The high intensity of the satellite line at $344 \mathrm{MHz}$ is easily understood by noting that each carbon atom affects the local field on the six $\mathrm{Mn}_{I I}$ atoms located in the corners of the $6 g$ octahedron. We will label those those $\mathrm{Mn}_{I I}$ atoms that form the octahedron hosting the carbon dopant as $\mathrm{Mn}_{I I_{-} \mathrm{C}}$, to differentiate from the $\mathrm{Mn}_{I I}$ sites that do not have carbon nearest neighbors.

The $84-\mathrm{MHz}$ frequency shift from $428 \mathrm{MHz}$ in $\mathrm{Mn}_{I I}$ sites of the pristine film down to $344 \mathrm{MHz}$ in the case of $\mathrm{Mn}_{5} \mathrm{Ge}_{3} \mathrm{C}_{0.2}$ reflects the carbon-induced decrease in the $\mathrm{Mn}_{I I}$ local field by $7.96 \mathrm{~T}$. In view of the fact that the main contribution to the hyperfine field was shown to arise from the Fermi contact term, and taking the hyperfine interaction constant $A=12 \mathrm{~T} / \mu_{B}$ [23]), the observed 7.96-T drop of hyperfine field implies a decrease in the magnetic moment of $\mathrm{Mn}_{I I_{-} \mathrm{C}}$ atoms of around $0.7 \mu_{B}$ i.e., by $20 \%$ with respect to the $\mathrm{Mn}_{I I}$ site in the pristine film. This is in good agreement with the previously calculated $24 \%$ decrease in the $\mathrm{Mn}_{I I}$ magnetic moment, which was attributed to hybridization between Mn $3 d$ and C $2 p$ orbitals [15]. Interestingly, the original NMR line from the unperturbed $\mathrm{Mn}_{I I}$ environments still persists around $420 \mathrm{MHz}$ (see Figs. 2 and 3), showing that some of the $\mathrm{Mn}_{I I}$ nuclei were not significantly affected by the presence of carbon. These are the $\mathrm{Mn}_{I I}$ sites that do not have carbon atoms in their nearest neighborhood. A miniscule downshift of the NMR frequency from 428 to $420 \mathrm{MHz}$, which indicates a slight drop of hyperfine field (from 40.56 to $39.8 \mathrm{~T}$ ) at the $\mathrm{Mn}_{I I}$ sites not having $\mathrm{C}$ as the nearest neighbor could be related to the decreased contribution transferred from further $\mathrm{Mn}_{I I_{-} \mathrm{C}}$ neighbors-which now have a reduced magnetic moment. In other words, the primary effect of carbon on the hyperfine field on $\mathrm{Mn}_{I I}$ nuclei is local and concerns those atoms that have carbon in their nearest-neighbor environment.

In contrast to the strong effect of carbon nearest neighbors on the ${ }^{55} \mathrm{Mn}$ hyperfine field in $\mathrm{Mn}_{I I}$ sites, the local fields on $\mathrm{Mn}_{I}$ sites are much less sensitive to the presence of carbon. Here, no distinct satellite line is resolved in the NMR spectrum corresponding to the magnetization pointing along the hexagonal $c$ axis. The main modification consists of a frequency downshift of the spectrum's gravity center and an increase in the $\mathrm{Mn}_{I} \mathrm{NMR}$ line half-width to $50 \mathrm{MHz}$ - much beyond the span of the original quadrupole-split line observed in the pristine film. This relatively weak effect of carbon on the $\mathrm{Mn}_{I}$ hyperfine field is to be expected, considering that the carbon-filled 2(b) interstitial positions are much more distant 


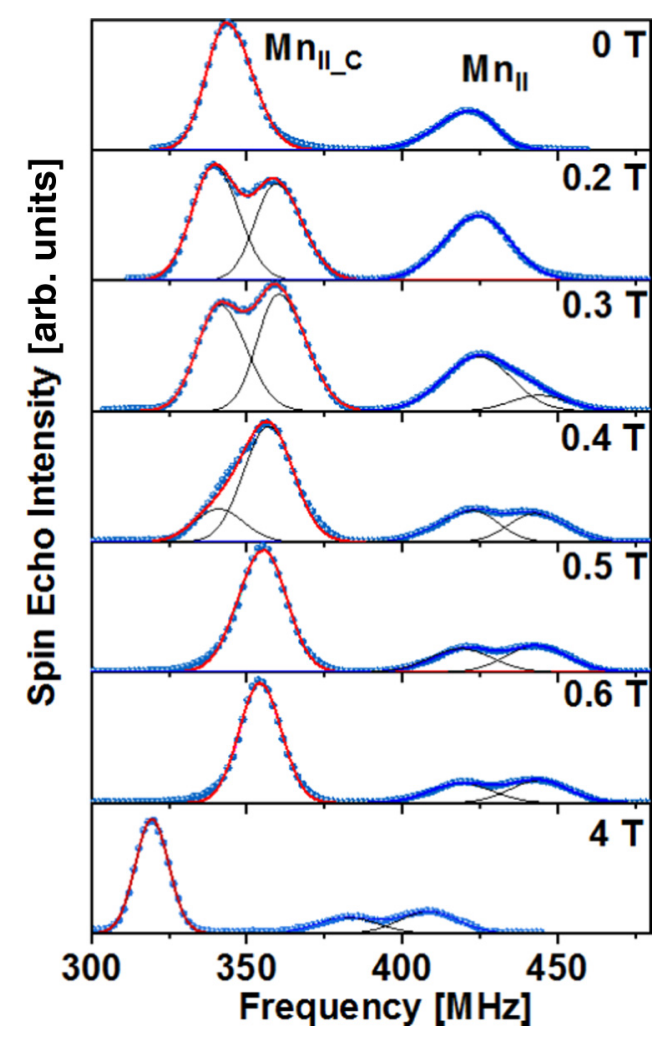

FIG. 4. ${ }^{55} \mathrm{Mn}$ NMR spectra recorded at $4.2 \mathrm{~K}$ from the $\mathrm{Mn}_{I I}$ sites in the $\mathrm{Mn}_{5} \mathrm{Ge}_{3} \mathrm{C}_{0.2}$ film at different values of the in-plane magnetic field. For details of spectrum decomposition, see the text.

from the $\mathrm{Mn}_{I}$ sites $\left(4.15 \AA\right.$ ) compared to $\operatorname{Mn}_{I I}(2.14 \AA)$. The significant broadening of the $\mathrm{Mn}_{I}$ NMR line reflects the distribution of $\mathrm{Mn}_{I}$ environments, having different numbers of $\mathrm{Mn}_{I I}$ neighbors with a reduced magnetic moment and will be investigated in further sections of this paper.

The zero-field NMR spectrum from the $\mathrm{Mn}_{5} \mathrm{Ge}_{3} \mathrm{C}_{0.2}$ film, supported by the experiment in the presence of the external magnetic field applied in the out-of-plane direction, provided information on the magnitude of ${ }^{55} \mathrm{Mn}$ hyperfine fields at three distinctly different types of Mn sites, more precisely on their component measured along the hexagonal $c$ axis. In order to investigate the in-plane component and estimate the local magnetic anisotropy in all three Mn environments, we performed a series of NMR experiments in the presence of an external magnetic field applied on the film plane.

\section{B. Hyperfine field anisotropy: NMR experiments in external magnetic field applied on the film plane}

Let us first consider the two kinds of $\mathrm{Mn}_{I I}$ sites, identified in the previous section as: (i) $\mathrm{Mn}_{I_{-} \mathrm{C}}$ that have carbon as a $\mathrm{nn}$ and (ii) $\mathrm{Mn}_{I I}$ that maintained their original nearest neighbors. Figure 4 presents the relevant portion of their NMR spectra, recorded with different values of the external magnetic field applied along the in-plane [210] direction.

\section{NMR line from the $\mathrm{Mn}_{I I}$ sites not having carbon nns}

As seen in Fig. 4, the application of an in-plane magnetic field leads to a rapid modification of the NMR lines from both

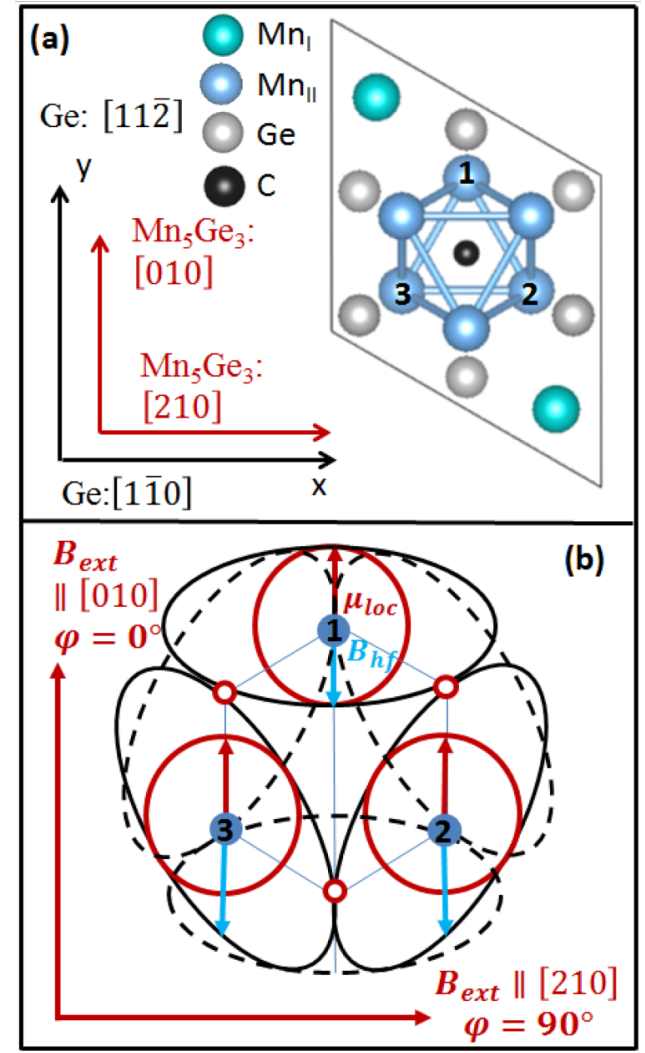

FIG. 5. (a) Projection of the atomic arrangement in the $\mathrm{Mn}_{5} \mathrm{Ge}_{3}$ unit cell on the (001) plane and the definition of the local coordinate system: The " $x$ " axis $\left(\phi=90^{\circ}\right)$ is along the [210] direction of $\mathrm{Mn}_{5} \mathrm{Ge}_{3}$ parallel to the [110] direction of the Ge substrate, the " $y$ " axis $\left(\phi=0^{\circ}\right)$ is along [010] in $\mathrm{Mn}_{5} \mathrm{Ge}_{3}$ and [11̄̄] in Ge. Numbers 1, 2 and 3 denote the $\mathrm{Mn}_{I I}$ atoms located on the same atomic plane $(z=1 / 4)$; (b) schematic presentation of the amplitude of in-plane hyperfine fields on the planes $z=1 / 4$ (solid black lines) and $z=3 / 4$ (dashed black lines).

types of $\mathrm{Mn}_{I I}$ environments. In the case of the $\mathrm{Mn}_{I I}$ sites that maintained their original nearest neighbors, the field-induced changes to the NMR spectrum resemble those reported for the $\mathrm{Mn}_{I I}$ NMR line in pristine $\mathrm{Mn}_{5} \mathrm{Ge}_{3}$ [21]. A single NMR line observed around $420 \mathrm{MHz}$ in the zero-field spectrum, broadens up in the presence of the in-plane field and develops a doublet structure. At around $0.5 \mathrm{~T}$, in-plane magnetic saturation is reached-the NMR spectrum shape does not change anymore and can be fitted with two broad Gaussian lines. This evolution of spectrum shape in the presence of the magnetic field is evidence for the in-plane anisotropy of hyperfine fields: The three $\mathrm{Mn}_{I I}$ atoms located on the same hexagonal plane of a unit cell [shown in the Fig. 5(a)] become magnetically nonequivalent.

To analyze the complex shape of the NMR spectrum observed in the in-plane saturated state, let us consider the atomic arrangement on the (001) basal plane of the $\mathrm{Mn}_{5} \mathrm{Ge}_{3}$ structure. As shown in Fig. 5(a), the epitaxial relationship is such that the [210] direction of $\mathrm{Mn}_{5} \mathrm{Ge}_{3}$ is parallel to the [110] direction of the Ge substrate-let us define this as the $x$ axis of our local Cartesian coordinate system. Consequently, the $y$ axis is parallel to [010] in $\mathrm{Mn}_{5} \mathrm{Ge}_{3}$ and [112̄] in Ge. The $z$ axis 


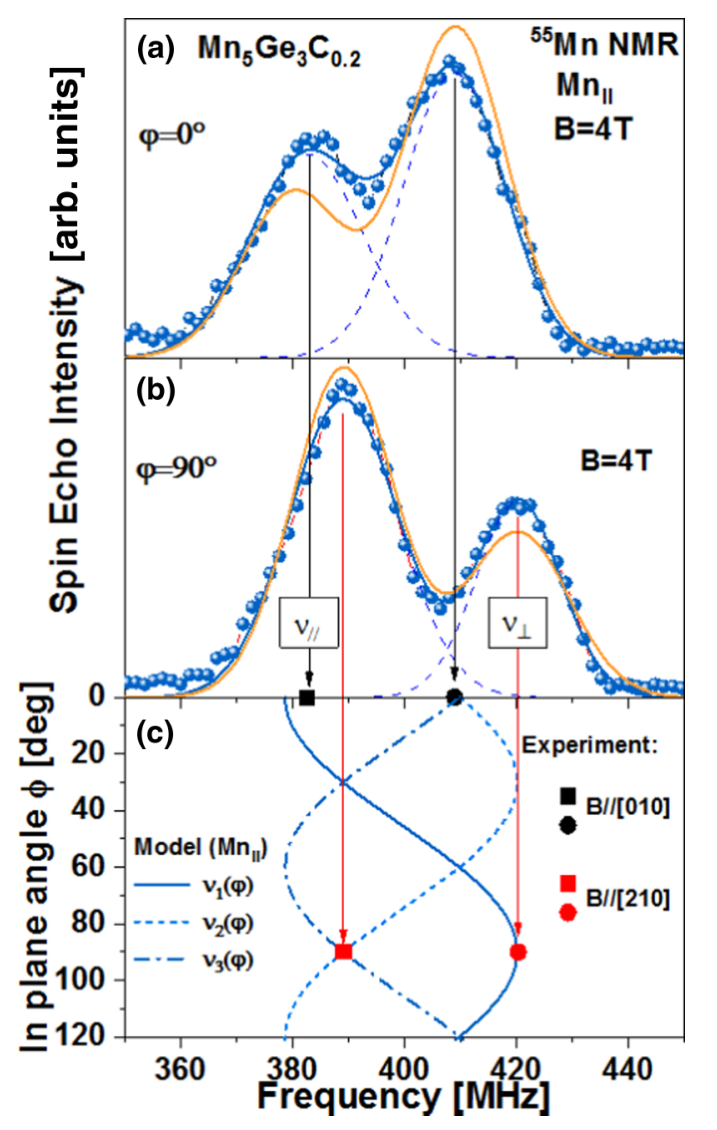

FIG. 6. (a) and (b) Blue spheres: ${ }^{55} \mathrm{Mn}$ NMR spectrum from the $\mathrm{Mn}_{I I}$ sites that have no carbon nearest neighbors, recorded at $4.2 \mathrm{~K}$ from the $\mathrm{Mn}_{5} \mathrm{Ge}_{3} \mathrm{C}_{0.2}$ film: (a) magnetic field of $4 \mathrm{~T}$ applied along the [010] direction $\left(\phi=0^{\circ}\right)$; (b) magnetic field of $4 \mathrm{~T}$ applied along the [210] direction $\left(\phi=90^{\circ}\right)$. Gaussian fit to the experimental spectrum (dashed blue lines). Solid orange line: Spectrum computed in frames of the model described in the text. (c) NMR frequency as a function of orientation of the in-plane field, computed for three nonequivalent $\operatorname{Mn}_{I I}$ positions $(n=1,2,3)$ located on the same atomic plane $(z=$ 1/4): $v_{n}(\phi)=v_{\|}+\Delta v \sin ^{2}[\phi+(n-1) 60]$.

is perpendicular to the film plane, i.e., along the hexagonal $c$ axis. The $\mathrm{Mn}_{I I}$ sites are hexagonally arranged on the two atomic planes within a unit cell. Three $\mathrm{Mn}_{I I}$ atoms (in Fig. 5 labeled as 1-3) are at $z=1 / 4$ and the remaining three $\mathrm{Mn}_{I I}$ atoms (here, not labeled) are at $z=3 / 4$, rotated by $60^{\circ}$ around the $c$ axis with respect to those located at $z=1 / 4$.

In the presence of an in-plane anisotropy, the spatial distribution of the hyperfine field amplitude at the hexagonally arranged $\mathrm{Mn}_{I I}$ sites is not spherical but resembles an ellipsoidas shown in Fig. 5(b). As the external magnetic field is rotated within the $x y$ plane, the cumulative NMR spectrum will vary depending on the direction of the external magnetic field since each particular $\mathrm{Mn}_{I I}$ site will contribute a signal at a different frequency. To estimate the magnitude of the in-plane anisotropy, we applied the magnetic field in two orthogonal directions: (i) along the $y$ axis (defining the azimuthal inplane angle as $\left.\phi=0^{\circ}\right)$ and (ii) along the $x$ axis $\left(\phi=90^{\circ}\right)$. The respective ${ }^{55} \mathrm{Mn}$ NMR spectra recorded in a field of $4 \mathrm{~T}$, i.e., in the magnetically saturated state, are presented in Figs. 6(a) and 6(b). In both experimental configurations, the

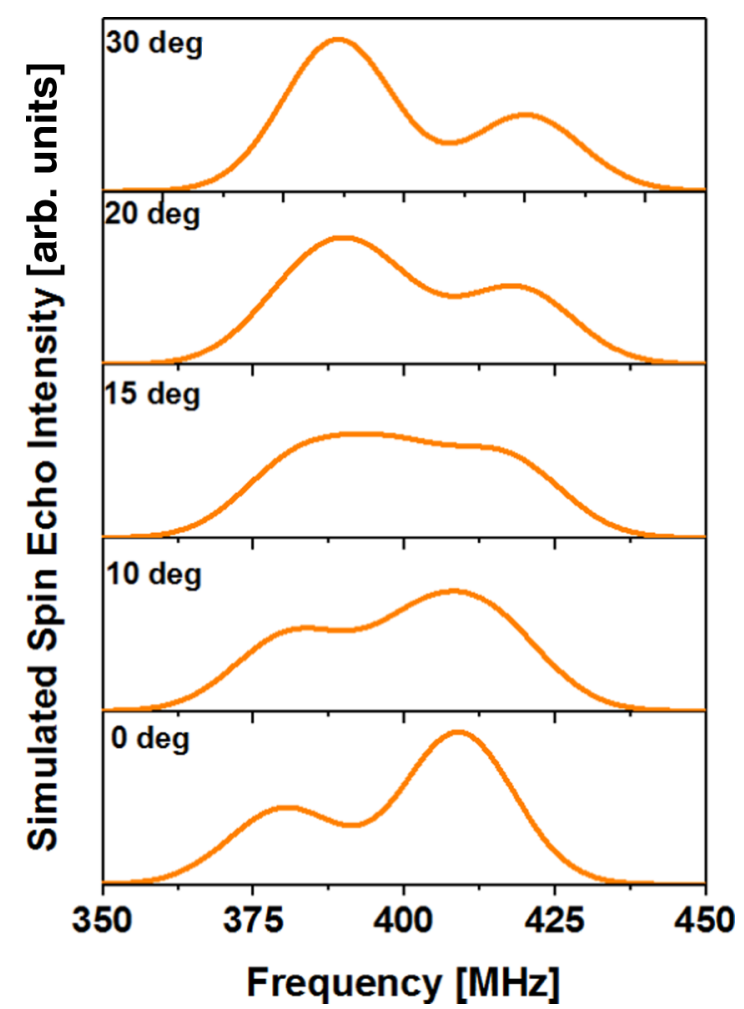

FIG. 7. The theoretical shape of the ${ }^{55} \mathrm{Mn}$ NMR spectra from the $\mathrm{Mn}_{I I}$ sites in the $\mathrm{Mn}_{5} \mathrm{Ge}_{3} \mathrm{C}_{0.2}$ film computed for the magnetic field of $4 \mathrm{~T}$ at various in-plane orientations with respect to the [010] direction.

NMR spectrum reveals a doublet structure, but the amplitude of a particular doublet component and its frequency differs strongly. To describe analytically the NMR frequency $v$ as a function of the azimuthal angle, we derived the formula (8), using the procedure described in Ref. [21]. For the case of a sample that is magnetically saturated in plane (i.e., the polar angle is $\theta=0^{\circ}$, and the azimuthal angle $\phi$ is fixed by the orientation of the external magnetic field), one obtains the following expression:

$$
v\left(\phi, B_{\text {ext }}\right)=v_{\|}+\Delta v \sin ^{2}[\phi+(n-1) 60]-\gamma B_{\text {ext }},
$$

where $v_{\|}$is the zero-field frequency corresponding to $\phi=$ $0^{\circ}$, whereas $\Delta v=v_{\|}-v_{\perp}$ is the frequency difference between the zero-field NMR frequency at $\phi=0^{\circ}\left(v_{\|}\right)$and $\phi=$ $90^{\circ}\left(v_{\perp}\right)$, representing the in-plane anisotropy of hyperfine fields. Numbers $n=1-3$ denote the $n$th $\mathrm{Mn}_{I I}$ nonequivalent position as defined in Fig. 6. The bottom panel in Fig. 6 presents the plot of Eq. (8) using the experimental values of the respective doublet frequencies at $\phi=0^{\circ}\left(v_{\|}=420 \mathrm{MHz}\right)$ and at $\phi=90^{\circ}\left(v_{\perp}=461.8 \mathrm{MHz}\right)$. The three frequency branches shifted by $60^{\circ}$ with respect to each other, represent the NMR signals from the three magnetically nonequivalent $\mathrm{Mn}_{I I}$ atoms located on one atomic plane. Superposition of NMR signals from these three branches determines the overall shape of ${ }^{55} \mathrm{Mn}$ NMR line from the $\mathrm{Mn}_{I I}$ sites that do not have carbon in the nearest neighborhood. This is shown in Fig. 7 presenting the cumulative NMR spectra computed as a sum of three components with the frequency given by the Eq. (8), each described by a Gaussian line having the same intensity 


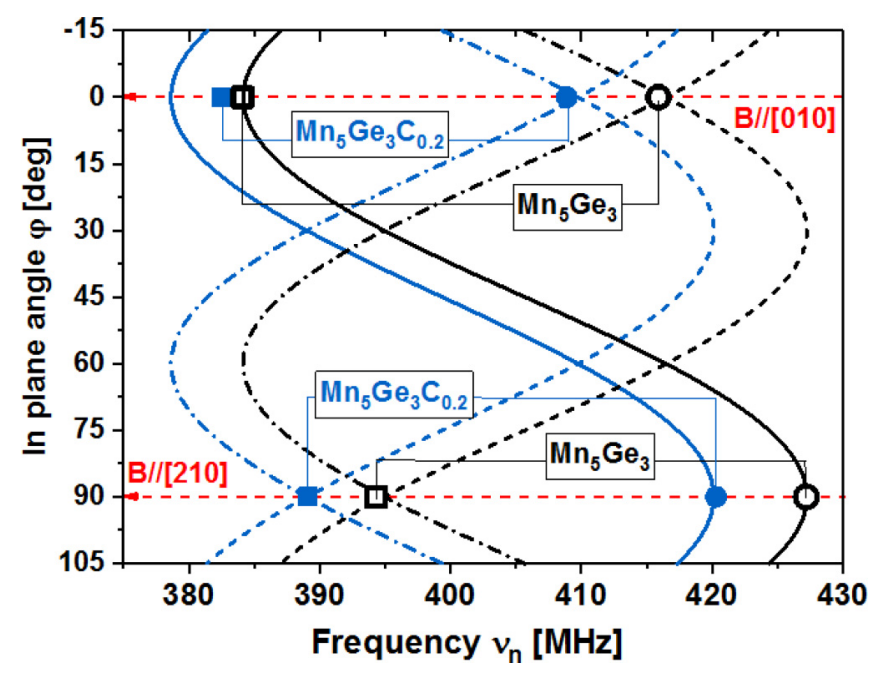

FIG. 8. Frequency branches of the NMR spectrum computed for three nonequivalent $\mathrm{Mn}_{I I}$ positions (solid line denotes $n=1$, dashed line denotes $n=2$, dashed dot line denotes $n=3$ ) as a function of orientation of the in-plane field in $\mathrm{Mn}_{5} \mathrm{Ge}_{3} \mathrm{C}_{0.2}$ (blue lines) and in the reference pristine $\mathrm{Mn}_{5} \mathrm{Ge}_{3}$ film (black lines): $v_{n}(\phi)=v_{\|}+$ $\Delta v \sin ^{2}[\phi+(n-1) 60]-\gamma B_{\text {ext }}$.

and a half-width. The computed spectra correctly reproduce the features of the experimental NMR spectra and illustrate how the shape of the NMR line varies when the direction of the in-plane field is changed. The experimental value of $\Delta v=41.8 \mathrm{MHz}$ corresponds to the hyperfine field difference between the two crystallographic directions amounting to $3.96 \mathrm{~T}$. To translate this difference into the anisotropy of the orbital moment, we apply Eq. (6), using the following values, calculated for the $\mathrm{Mn}_{I I}$ site in Ref. [5]: $\left\langle r^{-3}\right\rangle=2.18 a_{0}{ }^{-3}$, and, consequently, $A_{\text {orb }} \approx 27 T / \mu_{B}$. The resulting value of $\Delta \mu_{l}$ (the orbital moment anisotropy) equals $0.147 \mu_{B}$.

A similar in-plane anisotropy of hyperfine fields is also observed in a pristine $\mathrm{Mn}_{5} \mathrm{Ge}_{3}$ film with the following values of characteristic parameters: $v_{\|}=428$ and $\Delta v=43 \mathrm{MHz}$, corresponding to a hyperfine field difference of $4.08 \mathrm{~T}$ between the two crystallographic directions, and, thus, the anisotropy of the orbital moment is $\Delta \mu_{l}=0.151 \mu_{B}$. To illustrate this difference, Fig. 8 compares the plot of the resonance frequency given by Eq. (8) for $B_{\text {ext }}=4 \mathrm{~T}$ using the experimental values of $v_{\|}$and $\Delta v$ in $\mathrm{Mn}_{5} \mathrm{Ge}_{3} \mathrm{C}_{0.2}$ (blue lines) to the frequency plot for the pristine $\mathrm{Mn}_{5} \mathrm{Ge}_{3}$ film (black lines). As seen in Fig. 8, in the in-plane saturated film, only minor changes are observed with respect to the pristine film, showing a secondary effect of carbon on those $\mathrm{Mn}_{I I}$ atoms that do not have dopants in the nearest environment.

However, for the field values below saturation, the NMR lines in the C-doped film display somewhat different behavior compared to the pristine film. Figure 9 presents the NMR frequency of the two doublet components observed at $\phi=$ $90^{\circ}$ in $\mathrm{Mn}_{5} \mathrm{Ge}_{3} \mathrm{C}_{0.2}$ and in the pristine $\mathrm{Mn}_{5} \mathrm{Ge}_{3}$ film, plotted as a function of the in-plane field strength. In the pristine sample, the higher branch displays a steady frequency upshift: The magnetization is uniformly pulled away from the $c$ axis towards the $c$ plane, thus, increasing the in-plane magneticfield component. In contrast to that, the higher branch in the

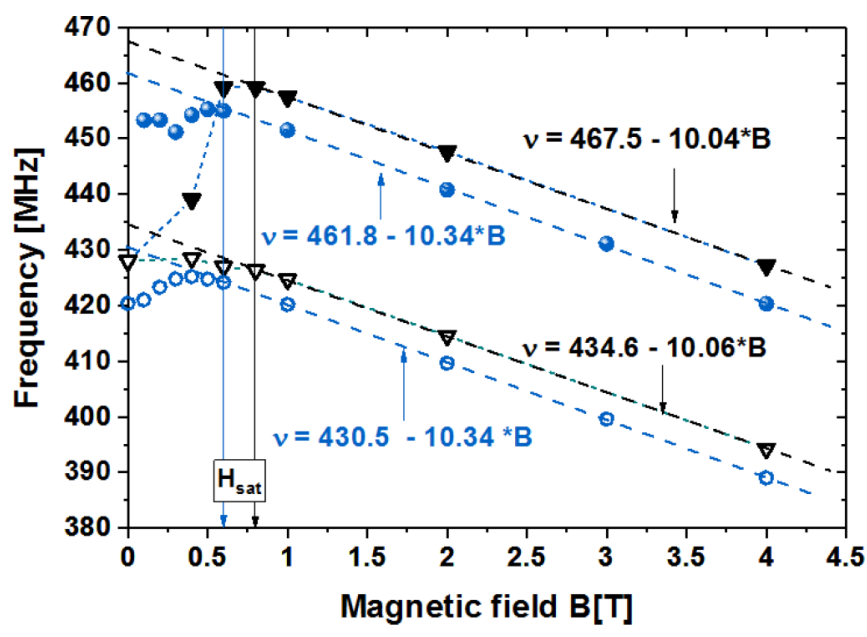

FIG. 9. NMR frequency of the two components of the ${ }^{55} \mathrm{Mn}$ NMR line from the $\mathrm{Mn}_{I I}$ sites not having carbon nearest neighbors, observed at $\phi=90^{\circ}$ in $\mathrm{Mn}_{5} \mathrm{Ge}_{3} \mathrm{C}_{0.2}$ (blue spheres) and in the reference pristine $\mathrm{Mn}_{5} \mathrm{Ge}_{3}$ film (black triangles), plotted as a function of the in-plane field strength.

C-doped film already reaches its maximum frequency at a very low external field and keeps a practically constant value with increasing field strength up to the in-plane saturation. This means that, in the $\mathrm{C}$-doped sample in the presence of even a weak in-plane field, a NMR signal is observed already from the regions with magnetization lying on the film plane. This effect is even more pronounced for the $\mathrm{Mn}_{I I_{-} \mathrm{C}} \mathrm{NMR}$ line as will be shown in the next section.

In the magnetically saturated state, the two samples reveal the same features: The frequency of all NMR lines decreases with a slope corresponding to the Mn gyromagnetic ratiothe external field is acting on the Mn nuclei in the opposite direction to the local field. The only difference is a slight decrease of the frequency distance between the two branches of the NMR signal in the C-doped film, resulting from the slight drop (from 4.08 to $3.9 \mathrm{~T}$ ) of the in-plane anisotropy of hyperfine fields as described above.

To conclude the observations on the $\mathrm{Mn}_{I I}$ sites not having carbon neighbors, we note that these Mn atoms experience only a slight effect of carbon doping, manifested by a small drop of the local field in the nucleus. The large local in-plane anisotropy of the orbital magnetic moment is still present and only slightly lower $\left(0.147 \mu_{B}\right)$ as compared to the $0.151 \mu_{B}$ in the pristine film. As a consequence, the local anisotropy of the orbital magnetic moment between the $c$ axis and the $c$ plane remains practically the same in these $\mathrm{Mn}_{I I}$ sites as in the pristine film.

\section{NMR line from the $\mathrm{Mn}_{I I_{-} \mathrm{C}}$ sites with carbon nns}

As seen in Fig. 4, in a field of 0.2 T, the NMR spectrum from the $\mathrm{Mn}_{I I_{-} \mathrm{C}}$ sites reveals a new component at $359 \mathrm{MHz}$. As the in-plane field strength increases, this component quickly gains intensity to become the only NMR line from the $\mathrm{Mn}_{I I_{-} C}$ sites when the in-plane magnetic saturation is reached at $0.5 \mathrm{~T}$. The frequency position of the line center and the relative intensity of the two components as a function of in-plane field strength are presented in Fig. 10. 


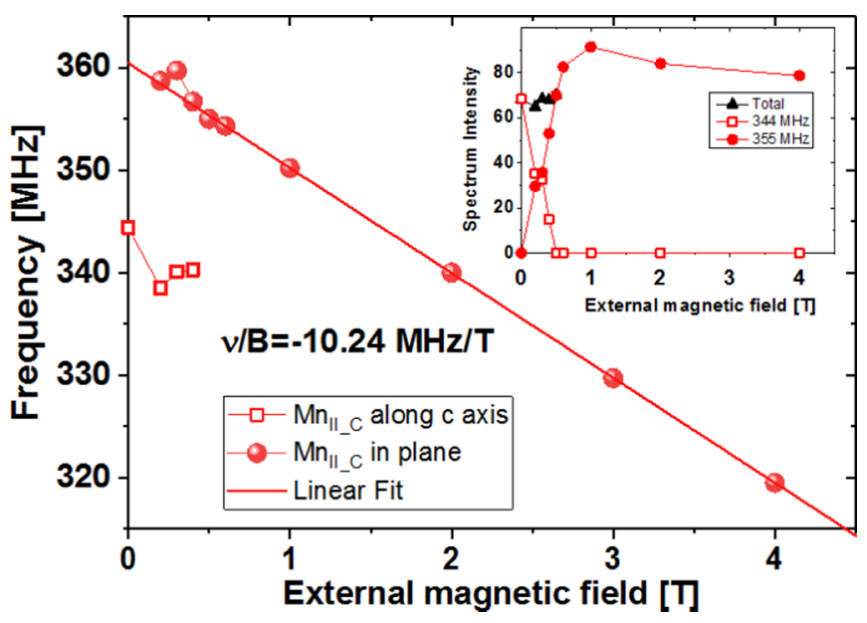

FIG. 10. ${ }^{55} \mathrm{Mn}$ NMR frequency of the two components of NMR spectrum from the $\mathrm{Mn}_{I I_{-} \mathrm{C}}$ sites of the $\mathrm{Mn}_{5} \mathrm{Ge}_{3} \mathrm{C}_{0.2}$ film as a function of the in-plane field. Open squares: NMR signal along the $c$ axis, spheres: NMR signal on the hexagonal plane. The inset: Intensities of the respective spectrum components as a function of the in-plane field strength and the total spectrum intensity (triangles) before the in-plane saturation.

The low-frequency component is a continuation of the original line at $344 \mathrm{MHz}$, which was previously identified as originating from the magnetic moments oriented along the $c$ direction. The second component persists beyond magnetic saturation and obviously corresponds to magnetization on the film plane. Below the in-plane saturation field strength, their cumulative intensity remains constant as shown in the inset of Fig. 10, meaning that the new component is growing at the expense of the original one. Clearly, in the presence of the magnetic field, some Mn moments rapidly switch to the in-plane orientation, and the process of magnetization reversal occurs through the increase of the volume of the in-plane oriented moments at the expense of those along the $c$ axis. This is consistent with the observations described above regarding the unperturbed $\mathrm{Mn}_{I I}$ atoms and is different from the situation in the pristine film where magnetic reorientation took place via the progressive incline of the entire magnetization towards the $c$ plane [21]. This difference can be understood considering the peculiarities of the domain structure reported in these films $[8,9]$. It has been shown that, in a pristine $\mathrm{Mn}_{5} \mathrm{Ge}_{3}$ film, a strong perpendicular anisotropy leads to the formation of a stripe domain structure in films thicker than $25 \mathrm{~nm}$. Since the material quality factor, defined as $Q=K_{u} / 2 \pi M_{S}^{2}$, is below 1

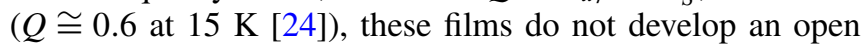
stripe domain structure but rather form flux closure caps at the film surface. In the case of the 300-nm-thick pristine film, the contribution of the flux closure caps is not significant and is not visible in the NMR spectra. However, in the case of the carbon-doped films, the bulk anisotropy is significantly lower [8], and the external in-plane field facilitates a rapid reorientation of a portion of magnetic moments towards the film plane, increasing the width of the Bloch walls and the volume of flux closure caps.

The resonance line corresponding to magnetization lying on the $c$ plane is shifted by $16.5 \mathrm{MHz}$ towards higher fre-

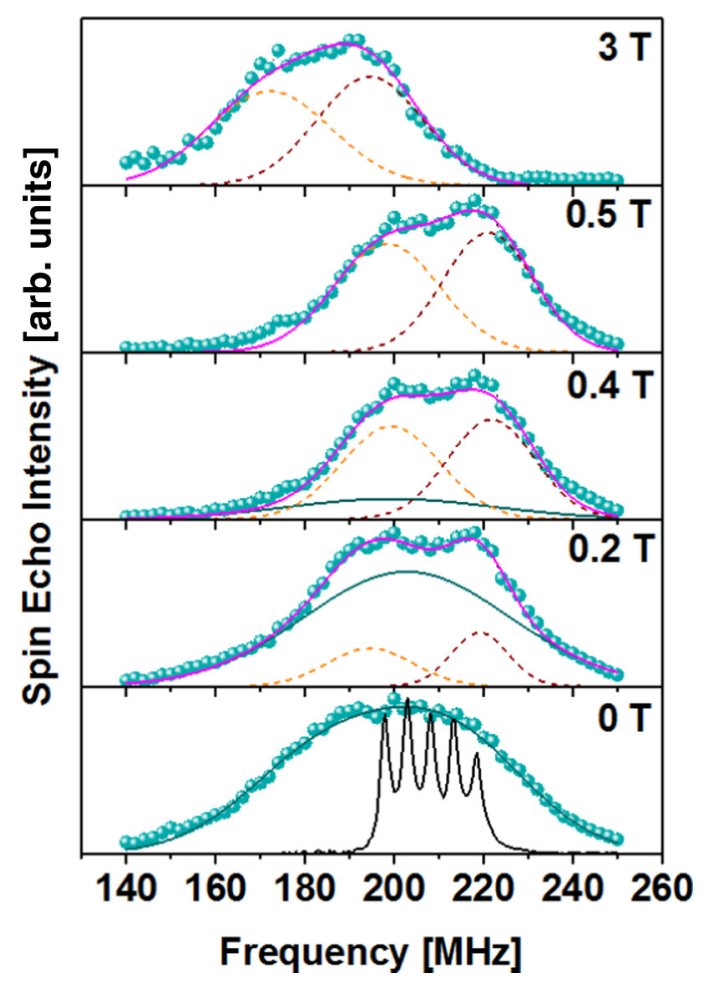

FIG. 11. Experimental ${ }^{55} \mathrm{Mn}$ NMR spectra from the $\mathrm{Mn}_{I}$ sites of the $\mathrm{Mn}_{5} \mathrm{Ge}_{3} \mathrm{C}_{0.2}$ film at $4.2 \mathrm{~K}$ at different values of in-plane magnetic fields (green bullets). Green solid line: NMR line for the out-of-plane oriented moments. Black solid line: Reference zerofield ${ }^{55} \mathrm{Mn}$ NMR spectrum in the pristine $\mathrm{Mn}_{5} \mathrm{Ge}_{3}$ film. Fit notation: Dashed orange and brown lines: Two components of the NMR spectrum for magnetization oriented in plane. Solid magenta line: A sum of the fit components.

quencies with respect to the zero-field NMR signal along the $c$ axis. This corresponds to the $1.56 \mathrm{~T}$ difference in the hyperfine field values between the two orientations. Consequently, the anisotropy of the orbital moment $\left(\Delta \mu_{l}\right)$ equals $0.058 \mu_{B}$. Interestingly, in the magnetically saturated state, the frequency position of the new component does not change upon in-plane rotation of the magnetic-field direction, meaning that the local field is isotropic in all the directions within the $c$ plane. This is in contrast to observations in the unperturbed $\mathrm{Mn}_{I I}$ sites and in the pristine film where the in-plane component of the hyperfine field on the $\mathrm{Mn}_{I I}$ site oscillated with a period of $60^{\circ}$.

To summarize, the presence of carbon as a near neighbor to the manganese atoms significantly modifies the local magnetic properties in the $\mathrm{Mn}_{I I}$ sites. The out-of-plane component of the hyperfine field on those $\mathrm{Mn}_{I I}$ sites that have carbon $\mathrm{nns}$, decreases by $7.96 \mathrm{~T}$ with respect to the pristine film, and the in-plane anisotropy of hyperfine field is lifted. The difference of hyperfine field strength between the $c$ direction and the $c$ plane is isotropic in plane and amounts to $1.56 \mathrm{~T}$ (resulting from the anisotropy of the orbital moment $\Delta \mu_{l}$ equal to $0.058 \mu_{B}$ ).

\section{NMR line from the $\mathrm{Mn}_{I}$ sites}

${ }^{55} \mathrm{Mn}$ NMR spectra from the $\mathrm{Mn}_{I}$ sites in the $\mathrm{Mn}_{5} \mathrm{Ge}_{3} \mathrm{C}_{0.2}$ film are shown in Fig. 11 (green spheres) at different values 
of the in-plane magnetic-field strength. Just as in the case of the pristine $\mathrm{Mn}_{5} \mathrm{Ge}_{3}$, the NMR spectrum from the $\mathrm{Mn}_{I}$ sites in $\mathrm{Mn}_{5} \mathrm{Ge}_{3} \mathrm{C}_{0.2}$ remained invariant with respect to the in-plane orientation of the external magnetic field.

As shown in the previous sections, the NMR signal at zero field is observed from the direction perpendicular to the film plane. To visualize the effect of carbon, the bottom panel presents also the zero-field ${ }^{55} \mathrm{Mn}$ NMR line from the $\mathrm{Mn}_{I}$ sites in the pristine $\mathrm{Mn}_{5} \mathrm{Ge}_{3}$ film, revealing a well-resolved quadrupolar splitting (black solid line). In the carbon-doped film, the broad linewidth and the smeared out quadrupolar structure indicate a significant modification of the crystal field due to inhomogeneities introduced by carbon. In the field of about $0.5 \mathrm{~T}$, the $\mathrm{Mn}_{5} \mathrm{Ge}_{3} \mathrm{C}_{0.2}$ film is fully saturated in plane, and the frequency span of the NMR line is narrower in comparison to the zero-field spectrum. The decreased linewidth of the NMR spectrum recorded in the in-plane saturated sample is consistent with the previously published observations in the pristine $\mathrm{Mn}_{5} \mathrm{Ge}_{3}$ film where the twice smaller quadrupolar splitting was reported in plane due to the out-of-plane orientation of the electric-field gradient [21].

In the case of the $\mathrm{Mn}_{5} \mathrm{Ge}_{3} \mathrm{C}_{0.2}$ film, the NMR spectrum recorded in the in-plane saturated film (above $0.5 \mathrm{~T}$ ) has a structure due to the reduced quadrupolar splitting. Therefore, the NMR spectrum can be decomposed into two broad Gaussian lines of equal linewidths, shown with the dotted lines in Fig. 11 This decomposition suggests the presence of two types of $\mathrm{Mn}_{I}$ environments, differing by the magnitude of the hyperfine fields. For the fields weaker than the in-plane saturation $(B<0.5 \mathrm{~T})$, a third component was needed in the fit, representing the NMR signal from the moments oriented out of plane. This component, which reproduces the zerofield NMR spectrum is known to represent the orientation perpendicular to the film plane and is shown with the green solid line in Fig. 11.

In an attempt to identify the two kinds of $\mathrm{Mn}_{I}$ environments revealed in the fit, the central frequencies of the two components identified in the spectrum from the in-plane orientation are plotted in the Fig. 12 as a function of the in-plane field strength, together with the field dependence of the $\mathrm{Mn}_{I}$ NMR line in the pristine $\mathrm{Mn}_{5} \mathrm{Ge}_{3}$ film, reported in Ref. [21].

Interestingly, the high-frequency component overlaps with the frequency position of the central line of the quadrupolar structure observed in the case of the pristine film. Therefore, it can be regarded as the signal from those $\mathrm{Mn}_{I}$ sites that do not feel the presence of carbon. Considering the local atomic coordination of the $\mathrm{Mn}_{I}$ site (see Fig. 1), we note that the nearest-neighbor positions are occupied by nonmagnetic Ge and manganese in the $\mathrm{Mn}_{I I}$ positions. Carbon is a more distant neighbor located at $4.15 \AA$ from the $\mathrm{Mn}_{I}$ site and is unlikely to directly influence the properties of manganese at this position. As shown in the previous sections, the effect of the carbon dopant is very local and limited to those $\mathrm{Mn}_{I I}$ sites where it enters as a nearest neighbor.

In view of this, it can be expected that the two $\mathrm{Mn}_{I}$ lines observed for the in-plane oriented magnetization represent two types of $\mathrm{Mn}_{I}$ sites having local environments populated by a different number of the possible $\mathrm{Mn}_{I I}$ neighbors: (i) $\mathrm{Mn}_{I I}$ with an unaltered first coordination zone and (ii) $\mathrm{Mn}_{I I_{-} \mathrm{C}}$ that

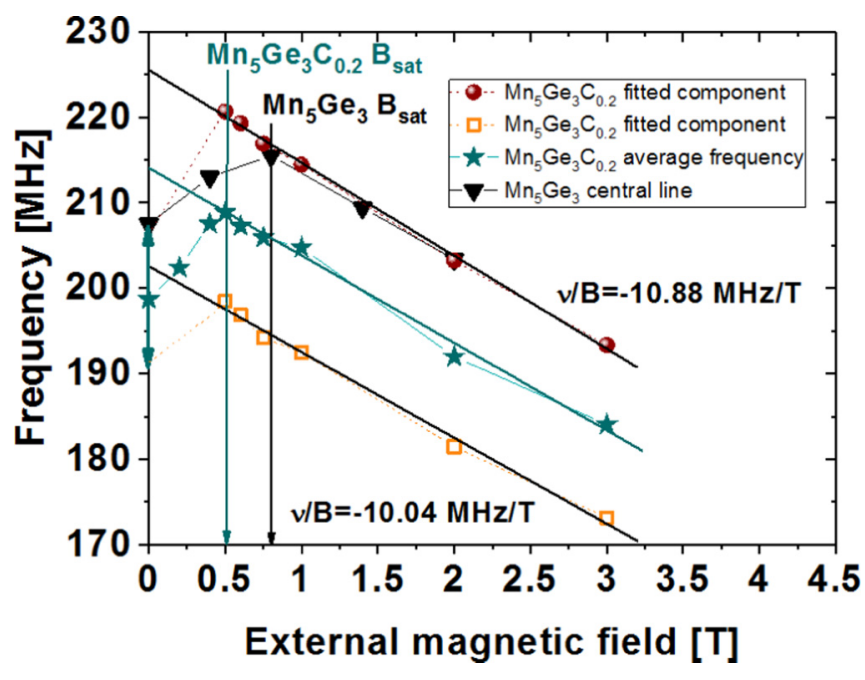

FIG. 12. Green stars: Central frequency of the experimental ${ }^{55} \mathrm{Mn}$ NMR spectrum from the $\mathrm{Mn}_{I}$ sites in the $\mathrm{Mn}_{5} \mathrm{Ge}_{3} \mathrm{C}_{0.2}$ film (computed as a line first moment). Brown spheres and orange squares: Spectrum components identified in Fig. 11; black triangles: Central line of the $\mathrm{Mn}_{I}$ quadrupolar quintet in the reference pristine $\mathrm{Mn}_{5} \mathrm{Ge}_{3}$. All plotted as a function of the in-plane magnetic-field strength.

have carbon as a nn. In other words, the effect of carbon on the hyperfine field in the $\mathrm{Mn}_{I}$ sites is indirect and occurs through a decrease in the transferred field from those $\mathrm{Mn}_{I I}$ neighbors that have a lower magnetic moment due to the carbon dopant in the vicinity. The frequency difference of $22 \mathrm{MHz}$ between the respective central frequencies indicates that the effect of the carbon dopant on the transferred field component of the hyperfine field in the $\mathrm{Mn}_{I}$ sites can be estimated at $2.1 \mathrm{~T}$.

\section{CONCLUSIONS}

The present NMR study provides strong evidence that carbon enters in the vicinity of the $6(\mathrm{~g})$ lattice sites of the $\mathrm{Mn}_{5} \mathrm{Ge}_{3}$ structure, confirming previous predictions that it occupies the $2 b$ voids formed by the $\mathrm{Mn}_{I I}$ octahedra. The $\mathrm{Mn}_{I I}$ atoms located in the corners of the host octahedron (here, labeled as $\mathrm{Mn}_{I I C}$ ) experience a serious modification of their properties. Table I gives the values of the out-of-plane component of hyperfine fields (i.e., the component along the hexagonal $c$ axis) for different $\mathrm{Mn}$ sites in $\mathrm{Mn}_{5} \mathrm{Ge}_{3} \mathrm{C}_{0.2}$, compared to pristine $\mathrm{Mn}_{5} \mathrm{Ge}_{3}$. It also lists the uniaxial anisotropy of hyperfine fields, defined as $\Delta B=B_{[001]}-B_{(001)}$, where $B_{(001)}$ is the hyperfine field measured in two orthogonal directions: [210] and [010], respectively, and the corresponding anisotropy of the orbital magnetic moment.

As seen in Table I, the absolute value of the out-of-plane component of the hyperfine field on the nuclei of the $\mathrm{Mn}_{I I_{-} \mathrm{C}}$ atoms is reduced from $40.56 \mathrm{~T}$ in the pristine film to $32.6 \mathrm{~T}$ in $\mathrm{Mn}_{5} \mathrm{Ge}_{3} \mathrm{C}_{0.2}$. The corresponding $0.7 \mu_{B}$ decrease in magnetic moment of $\mathrm{Mn}_{I I_{-} \mathrm{C}}$ atoms with respect to the $\mathrm{Mn}_{I I}$ site in the pristine film is in good agreement with the previously calculated 24\% decrease in the $\mathrm{Mn}_{I I}$ magnetic moment due to hybridization between $\mathrm{Mn} 3 d$ and C $2 p$ orbitals [15]. On the other hand, the $\mathrm{Mn}_{I I}$ atoms that do not have carbon as 
TABLE I. Hyperfine fields in different Mn sites of the $\mathrm{Mn}_{5} \mathrm{Ge}_{3} \mathrm{C}_{0.2}$ epitaxial film, their anisotropy and anisotropy of the orbital moment $\mu_{l}$ measured along the selected orthogonal directions, compared to the respective values in the pristine $\mathrm{Mn}_{5} \mathrm{Ge}_{3}$. The values of the hyperfine fields are expressed in teslas (T), and the orbital moment is given as the Bohr magneton $\mu_{B}$.

\begin{tabular}{|c|c|c|c|c|c|c|}
\hline \multirow[b]{2}{*}{ Site } & & \multirow{2}{*}{$\begin{array}{l}\text { Hyperfine field (T) } \\
\qquad(M \|[001])\end{array}$} & \multicolumn{2}{|c|}{$\Delta B=B(M \|[001])-B[M \|(001)](\mathrm{T})$} & \multicolumn{2}{|c|}{$\Delta \mu_{l}\left(\mu_{B}\right)$} \\
\hline & & & $B[001]-B[210]$ & $B[001]-B[010]$ & $\mu_{l}[001]-\mu_{l}[210]$ & $\mu_{l}[001]-\mu_{l}[010]$ \\
\hline & Pristine & -19.66 & 1.52 & 1.52 & 0.056 & 0.056 \\
\hline $\mathrm{Mn}_{I}$ & C doped & $-18.83^{\mathrm{a}}$ & $1.42^{\mathrm{a}}$ & $1.42^{\mathrm{a}}$ & 0.053 & 0.053 \\
\hline & Pristine & -40.56 & 4.08 & 0 & 0.151 & 0 \\
\hline $\mathrm{Mn}_{I I}$ & C doped & -39.8 & 3.96 & 0 & 0.147 & 0 \\
\hline $\mathrm{Mn}_{I I_{-} \mathrm{C}}$ & C doped & -32.6 & 1.56 & 1.56 & 0.058 & 0.058 \\
\hline
\end{tabular}

${ }^{a}$ Average hyperfine field and average hyperfine field anisotropy.

a nearest neighbor experience only a slight $(0.76 \mathrm{~T})$ drop of the hyperfine field, resulting from the decreased transferred field term from the more distant $\mathrm{Mn}_{I I_{C} \mathrm{C}}$ neighbors. A similar secondary effect of the reduced transferred hyperfine field is observed in the case of manganese located in the $4 d$ $\left(\mathrm{Mn}_{I}\right)$ sites for which there is evidence of two kinds of local environments in the in-plane saturated film that are attributed to a different number of $\mathrm{Mn}_{I I_{-} \mathrm{C}}$ neighbors.

In addition to decreasing the local magnetic moment of its immediate $\mathrm{Mn}_{I I}$ neighbors, the carbon dopant strongly modifies their local anisotropy. In the $\mathrm{Mn}_{I I_{-} \mathrm{C}}$ sites, the anisotropy of the orbital magnetic moment amounts to $0.058 \mu_{B}$ and does not vary with the azimuthal angle $\phi$. This is in clear contrast to the pristine film where it oscillates in plane between 0 and $0.151 \mu_{B}$ with a period of $60^{\circ}$. Such a strong effect of carbon is again limited only to the $\mathrm{Mn}_{I_{-} \mathrm{C}}$ sites that have carbon nearest neighbors, whereas the remaining $\mathrm{Mn}_{I I}$ sites as well as the $\mathrm{Mn}_{I}$ sites reveal anisotropy of the hyperfine fields not much different from the pristine film. According to the theoretical work of Bruno [16], in the itinerant ferromagnets, there exists a direct relationship between the anisotropy of the unquenched orbital momentum and the magnetocrystalline anisotropy. Bearing in mind that the orbital contribution to the hyperfine field is antiparallel to the dominant Fermi contact term, the fact that the absolute value of the overall hyperfine field is larger in plane for all Mn sites, means that the orbital term is larger along the $c$ axis. As a result of spinorbit interaction, in the scenario of the single-ion origin of magnetocrystalline anisotropy, this explains the $c$ axis as an easy magnetization direction. The decrease in macroscopic uniaxial anisotropy upon carbon doping [8] is due to the modified anisotropy of the orbital moment in the $\mathrm{Mn}_{I I_{-} \mathrm{C}}$ sites. In addition, in the pristine sample, there exists a sixfold inplane anisotropy, present also in residual form in the C-doped film. This sixfold in-plane anisotropy was macroscopically observed in the FM resonance experiment [25]. A direct carbon impact on magnetocrystalline anisotropy leads also to a strong modification of the crystal field, evidenced in our experiment by the vanishing quadrupolar splitting of the NMR line from the $\mathrm{Mn}_{I}$ site.
[1] C. Zeng, S. C. Erwin, L. C. Feldman, A. P. Li, R. Jin, Y. Song, J. R. Thompson, and H. H. Weitering, Appl. Phys. Lett. 83, 5002 (2003).

[2] M. Petit, L. Michez, C. E. Dutoit, S. Bertaina, V. O. Dolocan, V. Heresanu, M. Stoffel, and V. Le Thanh, Thin Solid Films 589, 427 (2015).

[3] A. Spiesser, F. Virot, L. A. Michez, R. Hayn, S. Bertaina, L. Favre, M. Petit, and V. Le Thanh, Phys. Rev. B 86, 035211 (2012).

[4] T. Nishimura, O. Nakatsuka, S. Akimoto, R. Hayn, W. Takeuchi, and S. Zaima, Microelectron. Eng. 88, 605 (2011).

[5] S. Picozzi, A. Continenza, and A. J. Freeman, Phys. Rev. B 70, 235205 (2004).

[6] R. P. Panguluri, C. Zeng, H. H. Weitering, J. M. Sullivan, S. C. Erwin, and B. Nadgorny, Phys. Status Solidi B 242, R67 (2010).

[7] Y. Tawara and K. Sato, J. Phys. Soc. Jpn. 18, 773 (1963).
[8] L. A. Michez, F. Virot, M. Petit, R. Hayn, L. Notin, O. Fruchart, V. Heresanu, M. Jamet, and V. Le Thanh, J. Appl. Phys. 118, 043906 (2015).

[9] R. Kalvig, E. Jedryka, P. Aleshkevych, M. Wojcik, W. Bednarski, M. Petit, and L. Michez, J. Phys. D: Appl. Phys. 50, 125001 (2017).

[10] J. M. Shaw, H. T. Nembach, and T. J. Silva, Phys. Rev. B 85, 054412 (2012).

[11] M. Gajdzik, C. Sürgers, M. T. Kelemen, and H. v. Löhneysen, J. Magn. Magn. Mater. 221, 248 (2000).

[12] A. Spiesser, I. Slipukhina, M.-T. Dau, E. Arras, V. Le Thanh, L. Michez, P. Pochet, H. Saito, S. Yuasa, M. Jamet, and J. Derrien, Phys. Rev. B 84, 165203 (2011).

[13] K. Momma and F. Izumi, J. Appl. Crystallogr. 44, 1272 (2011).

[14] A. Spiesser, V. Le Thanh, S. Bertaina, and L. A. Michez, Appl. Phys. Lett. 99, 121904 (2011).

[15] I. Slipukhina, E. Arras, P. Mavropoulos, and P. Pochet, Appl. Phys. Lett. 94, 192505 (2009). 
[16] P. Bruno, Phys. Rev. B 39, 865 (1989).

[17] R. Watson and A. J. Freeman, Phys. Rev. 123, 2027 (1961).

[18] A. Abragam and M. H. L. Pryce, Proc. Roy. Soc. (London) A 205, 135 (1951).

[19] S. Nadolski, M. Wojcik, E. Jedryka, and K. Nesteruk, J. Magn. Magn. Mater. 140-144, 2187 (1995).

[20] P. Panissod, M. Malinowska, E. Jedryka, M. Wojcik, S. Nadolski, M. Knobel, and J. E. Schmidt, Phys. Rev. B 63, 014408 (2000).

[21] R. Kalvig, E. Jedryka, M. Wojcik, G. Allodi, R. De Renzi, M. Petit, and L. Michez, Phys. Rev. B 97, 174428 (2018).
[22] E. Parthe and W. Jeitschko, Acta Crystallogr. 19, 1031 (1965).

[23] H. Akai, M. Akai, S. Blugel, B. Drittler, H. Ebert, K. Terakura, R. Zeller, and P. H. Dederichs, Prog. Theor. Phys. Suppl. 101, 11 (1990).

[24] L. A. Michez, A. Spiesser, M. Petit, S. Bertaina, J. F. Jacquot, D. Dufeu, C. Coudreau, M. Jamet, and V. Le Thanh, J. Phys.: Condens. Matter 27, 266001 (2015).

[25] Ch. E. Dutoit, V. O. Dolocan, M. Kuzmin, L. Michez, M. Petit, V. Le Thanh, B. Pigeau, and S. Bertaina, J. Phys. D: Appl. Phys. 49, 045001 (2016). 